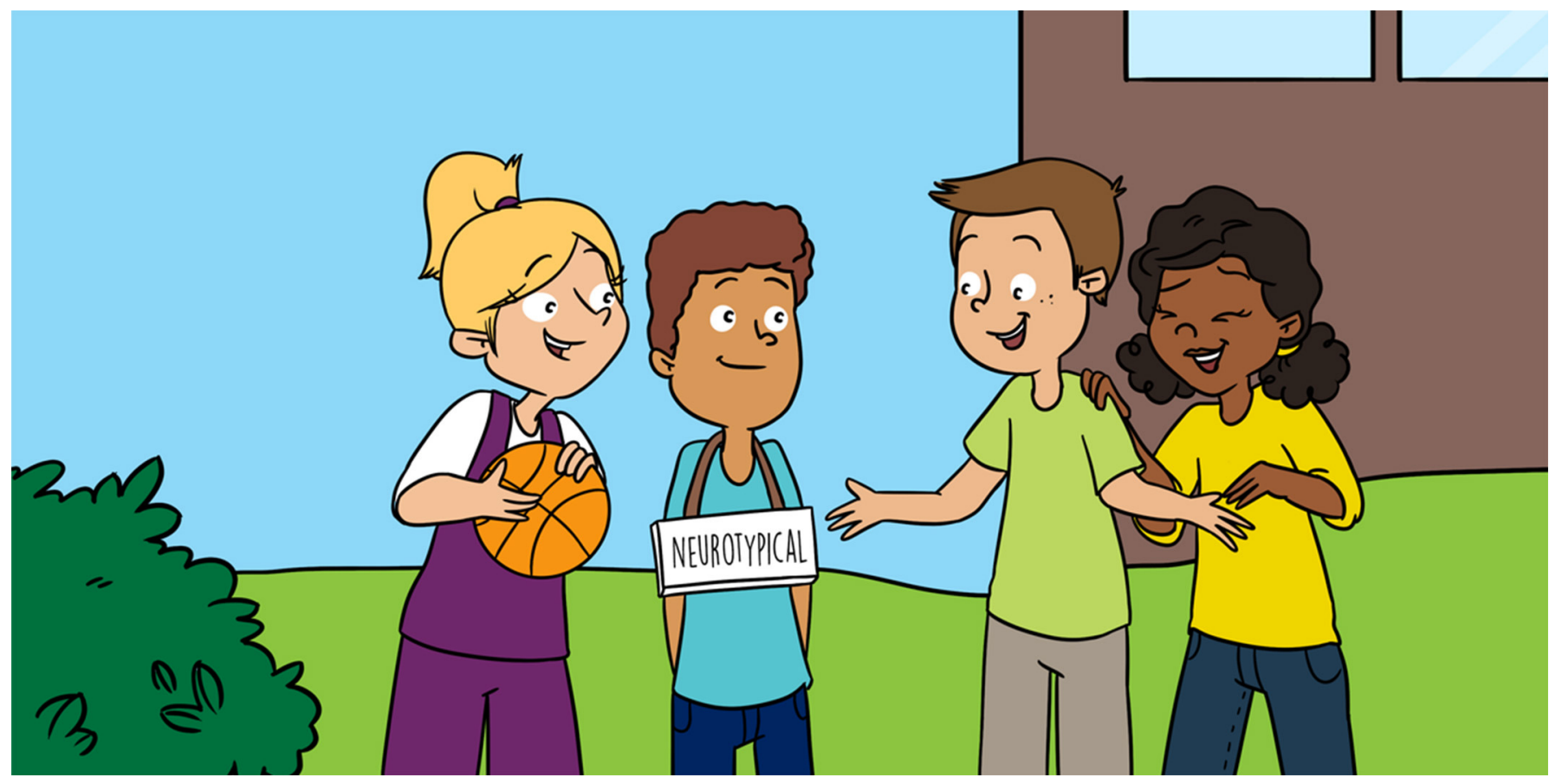

\title{
CAMOUFLAGING IN AUTISM
}

\section{Laura Hull * and Will Mandy}

Department of Psychology, University College London, London, United Kingdom

YOUNG REVIEWERS:

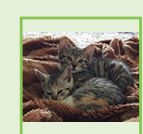

\section{HANNA}

AND JULE

AGES: $11-14$

\section{AUTISM}

A condition in which a person processes the world and understands others differently than most people do.

People are born with autism and it affects the way that they grow up. Some autistic people can find it hard to communicate or interact with others, and can feel overwhelmed in noisy, bright, or

busy situations.

Some people with autism try to hide aspects of their autism or develop ways to cope with it. This is known as camouflaging. Camouflaging involves finding ways around things that an autistic person finds difficult, hiding aspects of their autism, and trying to fit in with others. Overall, camouflaging might help some people to make friends, but it can be very exhausting and might even cause people harm.

\section{INTRODUCTION}

Do you know anyone who is autistic? The person might be a family member, a friend, someone in school, or even you! Autism means that a person can find it hard to interact or communicate with other people, and that he or she might experience the world in a different way. For example, an autistic person might find it difficult to ride on a train, because the noise can seem really loud and scary, but they might also be really interested in learning about animals and love cuddling with their pet. Everyone with autism is different, just like everyone without autism. 


\section{CAMOUFLAGING}

Finding ways to hide autism or make it less visible to other people, even if the autistic person is not aware of doing this.

\section{MASKING}

Hiding aspects of yourself or pretending to be like someone else. For example, a person might mask their autism by pretending to join in a game even if they do not understand the rules.

\section{COMPENSATION}

Finding ways around difficulties associated with autism. For example, an autistic person might compensate for not knowing which facial expressions to make by copying the facial expressions of a character in a TV show.

\section{ASSIMILATION}

Fitting in with other people. An autistic person might try to assimilate by always sitting next to the same person in class.
Some autistic people feel very different from everyone else, and might feel like they have to hide their autism or try to find ways to cope with the things they find difficult. We call this "camouflaging," because it is a bit like a chameleon changing the patterns on its skin to fit into the surroundings. The chameleon is still there, but it is trying to look like everything else around it. Examples of camouflaging in autism include forcing yourself to make eye contact with other people even if you do not want to, or thinking of a list of questions to ask every time you meet someone new, so you do not run out of things to say [1, 2].

Everyone-with or without autism-camouflages sometimes. For example, if you are feeling shy about meeting someone new, you might pretend to be really brave even though you do not feel it. But for some autistic people, camouflaging happens every time they talk to someone and this takes a lot of effort [3].

\section{LEARNING MORE ABOUT CAMOUFLAGING}

Autistic people have talked about camouflaging for a while [4], but doctors and researchers still do not know very much about it. No one knows whether camouflaging can help people or if it actually causes autistic people more problems. Researchers started to learn more about camouflaging by asking autistic people about their experiences of camouflaging or masking their autism $[3,5]$.

Autistic people reported that they camouflage their autism because they want to make friends and form connections with people, and because camouflaging can be helpful to them. Some people might camouflage their autism when they are at school or work, so that people do not treat them differently and they can concentrate on what they have to do. Some people camouflage their autism when they meet new people, so that they can make a good impression and maybe become friends. Some people will stop camouflaging when they get to know someone very well, if they think the person will accept them even when they act more autistic.

Several studies have found that autistic people use several different strategies to camouflage their autism. You can see a summary of the different types of strategies in Figure 1. For example, some people might practice making friendly faces in the mirror, because they find it hard to make those faces naturally. We call this "compensation," because these autistic people are compensating for a difficulty they have compared with non-autistic people. Another strategy is "masking," in which autistic people might hide how they are really feeling by, for instance, copying what someone else is talking about if they do not know what to say. Some autistic people also use strategies to try and fit in with other people, so they are not bullied or treated differently; we call this "assimilation." For example, these 
Figure 1

The main types of camouflaging strategies (compensation masking, and assimilation) and examples of these strategies.

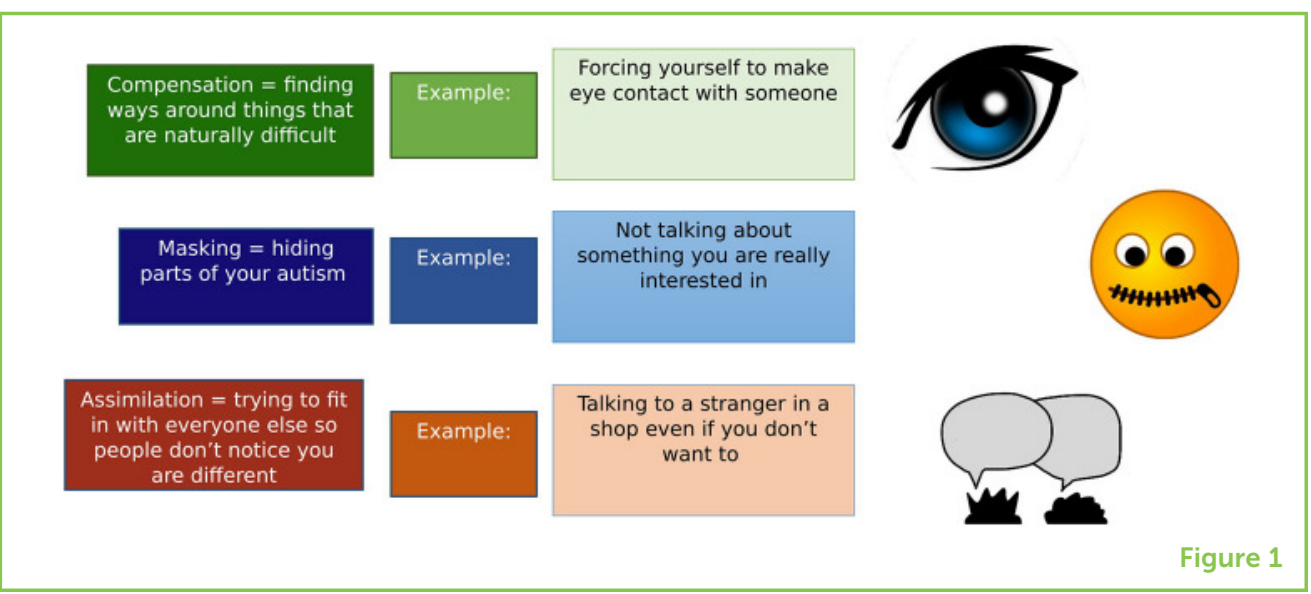

people might stand near other children in the playground, without playing with them.

\section{WHY IS IT IMPORTANT TO UNDERSTAND CAMOUFLAGING?}

Autistic people have lots of different feelings about camouflaging. For some people, camouflaging is helpful as it allows them to talk to people, go to school, and get a job without being treated differently because of their autism. But many other autistic people would like to be able to be themselves, and to make friends, without having to hide their autism.

Camouflaging takes a lot of effort. Hiding parts of yourself and pretending to be someone else can be really tiring. Many autistic people say that if they spend a whole day camouflaging at school or work, they need to come home and sit by themselves for a while to recover. One woman in a study said, "It is really exhausting!" [5]. Some autistic people also feel like they are being fake versions of themselves, and they want to be able to stop camouflaging so that their friends can get to know the "real" them.

Researchers can measure camouflaging in different ways. One way is to look at the difference between how someone feels and how they behave. If someone feels like they have a lot of autistic behaviors (such as finding unexpected changes hard to cope with), but other people do not notice this, they might be camouflaging their autism a lot [2]. Another way to measure camouflaging is by asking people how many camouflaging strategies they use, on a questionnaire called the Camouflaging Autistic Traits Questionnaire (CAT-Q [1]).

It is important for researchers and doctors to understand more about how people camouflage, because camouflaging might make people unwell. For example, some studies have found that people who camouflage more also have more mental health problems, such as 
depression or anxiety. It is important that we learn more about why these mental health problems happen, so we can help people to feel better. There are some situations in which camouflaging might be helpful (for example, in a school assembly where the autistic person has to sit quietly), but there are other situations in which camouflaging might make things harder (such as at a doctor's appointment, when you need to tell the doctor what is wrong). If autistic people can choose when to camouflage their autism, they might be happier and feel more like themselves.

\section{GENDER DIFFERENCES IN CAMOUFLAGING?}

Some autistic people and some researchers think that girls camouflage their autism more than boys do. This could be because girls feel more pressure to fit in with other people, or because people often do not realize that girls can have autism. If girls camouflage their autism more often, it could mean that it might be harder for doctors or teachers to realize when a girl is finding things difficult.

There has been a lot of research into gender differences in camouflaging over the last few years, and we still do not have a good answer! Some studies have found that girls show more of a difference between their autistic feelings and their behaviors, which might mean they camouflage more than boys do [2]. Other studies have found that girls and women report using more camouflaging strategies than boys do [1]. However, other studies have found that boys and men also camouflage frequently, which means that doctors and teachers should be aware that autistic boys might also find things hard [3].

We need to do more research in order to fully understand whether gender plays a role in camouflaging autism. At the moment, we know that all autistic people might camouflage their autism, and so it is important that we keep asking all autistic people about how camouflaging affects them.

\section{SUMMARY}

In this article, we described what camouflaging is, to help researchers and doctors understand what camouflaging is like for autistic people. Our article also suggests that, although there might be some benefits to camouflaging, such as making friends more easily, it can also be very harmful to autistic people. As many autistic people have said, it is important to make the rest of the world a friendlier place for autistic people, so they can be themselves and still be accepted. Once this happens, there will be no need for an autistic person to camouflage unless he or she wants to. 
Now that we know more about what camouflaging is, we want to learn more about how different people camouflage. In particular, are there differences in the ways that boys and girls use camouflaging techniques? Could these differences in how the genders use camouflaging affect whether or not their autism is diagnosed by a doctor? There is still a lot of research to do!

\section{ORIGINAL SOURCE ARTICLE}

Hull, L., Petrides, K. V., Allison, C., Smith, P., Baron-Cohen, S., Lai, M.-C., et al. 2017. "Putting on my best normal": social camouflaging in adults with autism spectrum conditions. J. Autism Dev. Disord. 47:2519-34. doi: $10.1007 / s 10803-017-3166-5$

\section{REFERENCES}

1. Hull, L., Mandy, W., Lai, M., Baron-Cohen, S., Allison, C., Smith, P., et al. 2018. Development and validation of the camouflaging autistic traits questionnaire (CAT-Q). J. Autism Dev. Disord. 49:819-33. doi: 10.1007/s10803-018-3792-6

2. Lai, M.-C., Lombardo, M. V., Ruigrok, A. N. V., Chakrabarti, B., Auyeung, B., Szatmari, P., et al., MRC AIMS Consortium. 2017. Quantifying and exploring camouflaging in men and women with autism. Autism 21:690-702. doi: $10.1177 / 1362361316671012$

3. Cage, E., and Troxell-Whitman, Z. 2019. Understanding the reasons, contexts and costs of camouflaging for autistic adults. J. Autism Dev. Disord. 49:1899-911. doi: 10.1007/s10803-018-03878-x

4. Bargiela, S., Steward, R., and Mandy, W. 2016. The experiences of late-diagnosed women with autism spectrum conditions: an investigation of the female autism phenotype. J. Autism Dev. Disord. 46:3281-94.

doi: $10.1007 / \mathrm{s} 10803-016-2872-8$

5. Hull, L., Petrides, K. V., Allison, C., Smith, P., Baron-Cohen, S., Lai, M.-C., et al. (2017). "Putting on my best normal": social camouflaging in adults with autism spectrum conditions. J. Autism Dev. Disord. 47:2519-34. doi: $10.1007 / s 10803-017-3166-5$

SUBMITTED: 09 July 2019; ACCEPTED: 11 November 2019;

PUBLISHED ONLINE: 27 November 2019.

EDITED BY: Eilidh Cage, Royal Holloway, University of London, United Kingdom

CITATION: Hull L and Mandy W (2019) Camouflaging in Autism. Front. Young Minds 7:129. doi: 10.3389/frym.2019.00129

CONFLICT OF INTEREST: The authors declare that the research was conducted in the absence of any commercial or financial relationships that could be construed as a potential conflict of interest. 

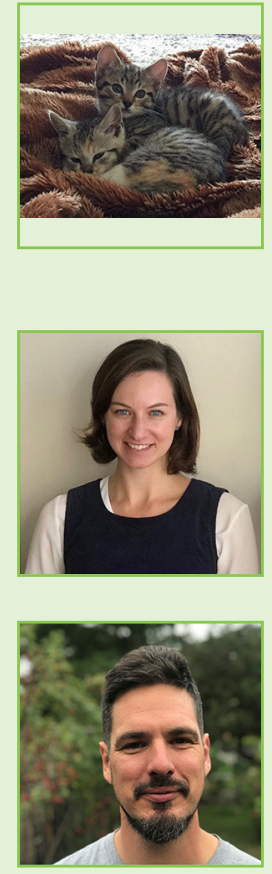

COPYRIGHT (c) 2019 Hull and Mandy. This is an open-access article distributed under the terms of the Creative Commons Attribution License (CC BY). The use, distribution or reproduction in other forums is permitted, provided the original author(s) and the copyright owner(s) are credited and that the original publication in this journal is cited, in accordance with accepted academic practice. No use, distribution or reproduction is permitted which does not comply with these terms.

\section{YOUNG REVIEWERS}

\section{HANNA AND JULE, AGES: 11-14}

We are sisters and both of us love to play with our cats. Our favorite subjects in school are art and maths.

\section{AUTHORS}

\section{LAURA HULL}

Laura Hull is a research psychologist who studies autism, gender, and social interaction. Her research is focused on improving outcomes for autistic people, including mental health, accessing support, and helping people feel good about their autism. *laura.hull.14@ucl.ac.uk

\section{WILL MANDY}

Will Mandy is a research and clinical psychologist. His research covers a wide range of topics, including autism and eating disorders, with recent research focused on helping people get an autism diagnosis, and supporting autistic children as they grow up and change schools. 